Sharif University of Technology
Scientia Iranica
SCIENTIA

\title{
Zero and nonzero normal fluxes of thermal radiative boundary layer flow of nanofluid over a radially stretched surface
}

\author{
N.A. Ramly, S. Sivasankaran and N.F.M. Noor* \\ Institutes of Mathematical Sciences, Faculty of Science, University of Malaya, 50603 Kuala Lumpur, Malaysia.
}

Received 22 December 2015; received in revised form 13 April 2016; accepted 4 March 2017

\author{
KEYWORDS \\ Nanofluid; \\ Axisymmetric flow; \\ Thermal radiation; \\ Stretching sheet; \\ Zero flux; \\ Boundary layer.
}

\begin{abstract}
The axisymmetric flow of a nanoparticles-saturated fluid with existence of thermic radiation over a stretched sheet is investigated. The effects of zero (passive control) as well as nonzero fluxes (active control) of nanoparticles on the plate on distributions of temperature and volumetric fraction of nanoparticles are investigated together comparatively. Through the supposition of boundary layer, the Navier-Stokes equations are simplified hence converted into non-dimensional form by similarity transformation. A shooting technique is engaged to deal with the emerging nonlinear system of ordinary differential equations numerically in MATLAB software. Several distributions of velocity, thermal energy, and volumetric fraction of nanoparticles under zero/nonzero normal flux are graphically demonstrated. The impact of the parameters on the reduced coefficient of skin friction, $\mathrm{Nu}_{r}$ and $\mathrm{Sh}_{r}$, are also investigated. The presence of thermic radiation under consideration of both zero and nonzero normal fluxes has significant effects on the intensification of the flow heat transfer. Thermophoresis enhances the heat conductivity performance in the case of zero fluxes of nanoparticles.

(C) 2017 Sharif University of Technology. All rights reserved.
\end{abstract}

\section{Introduction}

Industry fields such as manufacturing, health care, transportation, energy, and nuclear system have advanced with the rapid industrial progress in the past few years. One of the important elements that has been applied in such industries is cooling/heating process, which can now be enhanced by using nanofluid technology. Nanofluid was firstly utilized by Choi and Eastman [1] in 1995. Nowadays, this technology is widely used. According to Gorla and Kumari [2], nanofluid is now being developed for medical applications. On the other hand, Noghrehabadi et al. [3] stated

\footnotetext{
*. Corresponding author.

E-mail address: drfadiya@um.edu.my (N.F.M. Noor)
}

that the thermal energy transfer rate of nanofluid flows in the neighborhood of a stretched sheet thin layer was important as it was utilized in the application sectors of material science, chemical and engineering. Freeforced convection in nanofluid boundary layer flow also plays an imperative role in the fields of science and engineering [4]. This shows that nanofluid has had a major implication in many industries from the past decades up to now. In 2006, Buongiorno [5] developed a mathematical model for convective transport of nanofluid by considering seven slip mechanisms.

The axisymmetric flow model along a stretched plate with existence of partial slip at the boundary was studied by Ariel [6] and he provided an analytical result for the governing equations with nonlinearity. After that, a series of analytical solutions for time-dependent viscous fluid flow with heat transfer resulting from a radially stretched surface was retrieved by Sajid et al. [7]. 
Grosan and Pop [8] theoretically investigated axisymmetric free-forced convection water-based flow along a standing cylinder in the presence of copper nanoparticles with a fixed surface temperature and external flow. The study on stretching/shrinking movement in a mixed convection of both assisting and opposing flows along a vertical cylinder was done by Lok et al. [9]. Recently, there have been many studies about magnetohydrodynamics (MHD) effect on axisymmetric flow. For example, Vajravelu et al. [10] investigated MHD axisymmetric heat generating incompressible flow with constant temperature over a stretched cylinder with thermal conductivity as variable.

A form of electromagnetic radiation that is emitted by all objects above absolute zero is called thermal radiation. Das et al. [11] stated that the thermal radiation reaction played a vital part in physics and engineering applications as well as in industries, where the regulation of the heat transfer was needed. Sajid and Hayat [12] studied the thermic radiation impact on the energy equation of a boundary layer flow along a surface with exponential stretching velocity. Siddiqa et al. [13] concluded that the thermal radiation contributed to delaying the fluid separation as well as the enhancement in heat transfer. They also concluded that an irregular horizontal surface transferred heat better than a smooth horizontal surface. Later, Ul Haq et al. [14] focused on the simultaneous consequences of thermal slip and displacement gradient near a stagnation point in MHD nanofluid flow towards a horizontally stretchable surface. Abdul Hakeem et al. [15] used thermal radiation to determine the influence of a partial slip within boundary layer of a hydromagnetic flow along a porous stretchable surface. Later, Abdul Hakeem et al. [16] examined MHD second-order slipped nanofluid flow with heat-transfer convection and thermal radiation along a stretching/shrinking surface.

The boundary-layer nanofluid flow problem over a stretchable plate has been analytically resolved by Hassani et al. [17]; they also inspected the response of Brownian random motion with thermophoretic force simultaneously. Makinde and Aziz [18] numerically solved the nanoparticles-saturated flow due to a linearly stretched plate using the convective condition of heating instead of a stationary temperature or a stationary heat flux at boundary of the surface. Then, Mustafa et al. [19] attained an analytical result for heat transport of a steady nanofluid flow within the whereabouts of a point of stagnation along a stretchable plate. A study of influence of different volumetric fractions of nanoparticles on a bidirectional flow of water-based nanoparticles within the boundary layer of an exponentially stretchable sheet was done by Nadeem et al. [20]. Noreen Sher Akhbar et al. [21] discussed a flow of suspended fluid in carbon nanotubes in the vicinity of a stagnant point over a stretched plate under the existence of hydrodynamic slip coupled with a convective boundary condition by using homogenous model. Currently, Ganesh et al. [22] have performed a comparative study on the flow of $\mathrm{Al}_{2} \mathrm{O}_{3}$ and $\gamma-\mathrm{Al}_{2} \mathrm{O}_{3}$ nanoparticles dispersed in different base fluids along a stretching plate.

The utilization of nanofluid leads to a formation of flux in the vicinity of the surface. In the current work, the mathematical model by Mustafa et al. [23] is extended to include radiation effect due to thermal heating on the axisymmetric flow saturated with nanoparticles. The zero normal mass flux at the wall proposed in $[24,25]$ is also incorporated in this upgraded model with the exception that, this time, the effects of both passive and active controls of nanoparticles (zero and nonzero normal fluxes) are analyzed together for comparison purpose, especially to investigate flow circumstances of the two situations for better heat transfer.

\section{Formulation of problem}

The time-independent laminar incompressible nanofluid flow along an oblique surface aligned with the $r \theta$ plane, as shown in Figure 1, is considered. The fluid mixture is in thermal state of equilibrium and no hydrodynamic slip bounded by the suspended nanoparticles and the based-fluid is assumed. The nanometer-size particles are presumed to be similar and behave like the base fluid molecules. The system of cylindrical coordinates is chosen as $(r, \theta, z)$. The fluid stays in the upper half space of the vertical axis, where $z \geq 0$. Along the spiral direction, the surface is stretched outward on the circular plane with its velocity in terms of power-law distribution. $T_{w}$ is the plate temperature and kept constant. At the wall, the nanoparticles

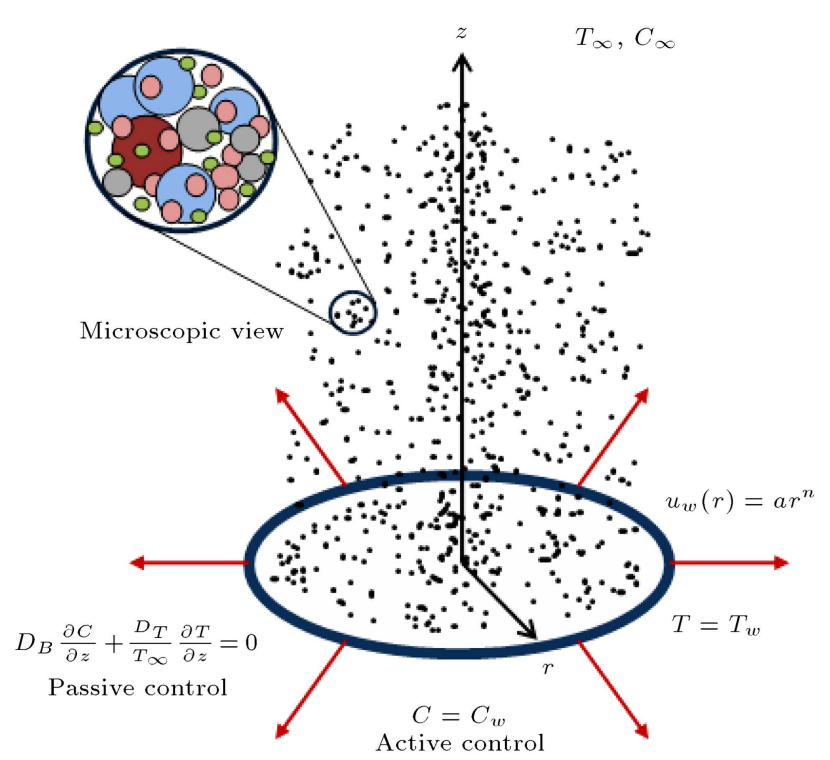

Figure 1. Geometry of the model. 
are considered to have zero normal flux and nonzero normal flux towards the surface in two different occasions. In the stream, the surrounding value of temperature is indicated by $T_{\infty}$, while the surrounding value of volumetric fraction of nanoparticles is denoted by $C_{\infty}$.

Therefore, the governing equations under the ordinary assumptions of boundary layer simplification for continuity, conservative momentum, energy, and volumetric fraction of nanoparticles can be pointed as in [23], except for the last term of Eq. (3), which is an additional expression for thermal radiation:

$$
\begin{aligned}
& \frac{\partial u}{\partial r}+\frac{u}{r}+\frac{\partial w}{\partial z}=0 \\
& u \frac{\partial u}{\partial r}+w \frac{\partial u}{\partial z}=\nu_{f} \frac{\partial^{2} u}{\partial z^{2}} \\
& u \frac{\partial T}{\partial r}+w \frac{\partial T}{\partial z}=\frac{k}{\rho c_{p}} \frac{\partial^{2} T}{\partial z^{2}} \\
& -\tau\left[D_{B} \frac{\partial C}{\partial z} \frac{\partial T}{\partial z}+\frac{D_{T}}{T_{\infty}}\left(\frac{\partial T}{\partial z}\right)^{2}\right]-\frac{1}{\rho c_{p}} \frac{\partial q_{r}}{\partial z} \\
& u \frac{\partial C}{\partial r}+w \frac{\partial C}{\partial z}=D_{B} \frac{\partial^{2} C}{\partial z^{2}}+\frac{D_{T}}{T_{\infty}} \frac{\partial^{2} T}{\partial z^{2}}
\end{aligned}
$$

where $u$ and $w$ are velocity vector components in $r$-direction and $z$-direction, respectively; $\nu_{f}$ is fluid kinematic viscosity; $\rho$ fluid density; $k$ fluid thermal conductivity; $c_{p}$ specific heat with constant pressure; $\tau$ proportion of effective capacity of heat in nanoparticlematerial to that of the fluid; $D_{B}$ coefficient of Brownian random motion; and $D_{T}$ coefficient of thermophoretic diffusion. The conditions at boundary for this considered problem are:

$$
\begin{aligned}
& \text { At } z=0 \text {, } \\
& u=u_{w}(r)=a r^{n}, \quad T=T_{w}, \\
& \begin{cases}C=C_{w} & \text { (active control) } \\
D_{B} \frac{\partial C}{\partial z}+\frac{D_{T}}{T_{\infty}} \frac{\partial T}{\partial z}=0 & \text { (passive control) }\end{cases}
\end{aligned}
$$

as $z \rightarrow \infty$,

$$
u \rightarrow 0, \quad T \rightarrow T_{\infty}, \quad C \rightarrow C_{\infty},
$$

where $a>0$ denotes the stretching coefficient, $a<0$ the shrinking coefficient, and $n>0$ the index of powerlaw. Note that Condition (5), which combined both active and passive controls of nanoparticles at two different occasions, is new here.

On imposing Rosseland approximation for heat flux radiation $q_{r}$ [14], we can get:

$$
q_{r}=-\frac{4 \sigma_{1}}{3 k^{*}} \frac{\partial T^{4}}{\partial z}
$$

where $\sigma_{1}$ is the constant of Stefan-Boltzmann and $k^{*}$ the coefficient of mean absorption. Presuming sufficiently small temperature differences within the flow, we can express $T^{4}$ as a temperature linear function by employing Taylor's series and ignoring the higher order terms:

$$
T^{4} \cong 4 T_{\infty}^{3} T-3 T_{\infty}^{4}
$$

Following [12] for Cartesian plane coordinate, Eq. (3) can be rewritten in cylindrical coordinates by using Eqs. (7) and (8), which yields:

$$
\begin{aligned}
\frac{u \partial T}{\partial r} & +w \frac{\partial T}{\partial z}=\frac{k}{\rho C_{p}} \frac{\partial^{2} T}{\partial z^{2}} \\
& +\tau\left[D_{B} \frac{\partial C}{\partial z} \frac{\partial T}{\partial z}+\frac{D_{T}}{T_{\infty}}\left(\frac{\partial T}{\partial z}\right)^{2}\right] \\
& +\frac{16 T_{\infty}^{3} \sigma_{1}}{3 \rho C_{p} k^{*}} \frac{\partial^{2} T}{\partial z^{2}}
\end{aligned}
$$

where the third term of the RHS of Eq. (9) denotes the present additional expression of thermal radiation.

Now, the governed expressions (Eqs. (2)-(4)) are possibly converted to a list of nonlinear ODEs by means of proposing the similarity transformation and variables without unit dimension:

$$
\begin{aligned}
& u=a r^{n} f^{\prime}(\eta), \\
& w=a r^{(n-1) / 2} \sqrt{\frac{\nu_{f}}{a}}\left(\frac{n+3}{2} f(\eta)+\frac{n-1}{2} \eta f^{\prime}(\eta)\right), \\
& \eta=\sqrt{\frac{a}{\nu_{f}}} r^{(n-1) / 2} z, \quad \theta=\frac{T-T_{\infty}}{T_{w}-T_{\infty}}, \\
& \begin{cases}\phi=\frac{C-C_{\infty}}{C_{\infty}} & \text { (active control), } \\
\phi=\frac{C-C_{\infty}}{C_{w}-C_{\infty}} & \text { (passive control). }\end{cases}
\end{aligned}
$$

Using Eq. (10), the following ODE is obtained:

$$
\begin{aligned}
& f^{\prime \prime \prime}+\frac{n+3}{2} f f^{\prime \prime}-n f^{\prime} 2=0 \\
& \frac{1}{P r}\left(1+\frac{4}{3} R d\right) \theta^{\prime \prime}+\frac{n+3}{2} \theta^{\prime} f+N b \theta^{\prime} \phi^{\prime}+N t \theta^{2}=0 \\
& \phi^{\prime \prime}+\frac{n+3}{2} \operatorname{Sc} f \phi^{\prime}+\frac{N b}{N t} \theta^{\prime \prime}=0
\end{aligned}
$$

subject to: 


$$
\begin{aligned}
& f(0)=0, \quad f^{\prime}(0)=1, \quad \theta(0)=1, \\
& \left\{\begin{array}{l}
\phi(0)=1 \\
N b \phi^{\prime}(0)+N t \theta^{\prime}(0)=0 \quad \text { (pastive control) },
\end{array}\right. \\
& f^{\prime}(\infty) \rightarrow 0, \quad \theta(\infty) \rightarrow 0, \quad \phi(\infty) \rightarrow 0 .
\end{aligned}
$$

In the formerly stated equations, $\operatorname{Pr}=\mu c_{p} / k$ represents the Prandtl number, $R d=4 T_{\infty}{ }^{3} \sigma^{1} / k^{*} k$ the parameter of thermal radiation, $\mathrm{Sc}=\nu_{f} / D_{B}$ the Schmidt number, $N b=\rho C_{p} D_{B} C_{\infty} / \rho C_{f} \nu_{f}$ the parameter of Brownian random motion, and $N t=\rho C_{p} D_{T}\left(T_{w}-\right.$ $\left.T_{\infty}\right) / \rho C_{f} T_{\infty} \nu_{f}$ the parameter of thermophoresis. We use variable (10) to obtain the dimensionless form of the coefficient of skin friction, $C_{f}$, for friction at the local point on the surface between solid and fluid in relative motion, the Nusselt number, $\mathrm{Nu}$, for heat transfer of the moving fluid at the local point of the surface, and the Sherwood number, Sh, for the volumetric fraction of nanoparticles distribution at the local point within the fluid flow, where:

$$
\begin{aligned}
C_{f} & =\frac{\mu_{f}\left(\frac{\partial u}{\partial z}\right)_{z=0}}{\rho_{f} u_{w}^{2}}, \\
\mathrm{Nu} & =\frac{-r\left(\frac{\partial T}{\partial z}\right)\left(\frac{16 T_{\infty}{ }^{3} \sigma_{1}}{3 k^{*}} \frac{\partial T}{\partial z}\right)_{z=0}}{k\left(T_{w}-T_{\infty}\right)}, \\
\mathrm{Sh} & =\frac{-r\left(\frac{\partial C}{\partial z}\right)_{z=0}}{C_{w}-C_{\infty}},
\end{aligned}
$$

can be restated into the following forms:

$$
\begin{aligned}
& \operatorname{Re}_{r}^{1 / 2} C_{f}=f^{\prime \prime}(0), \\
& \operatorname{Re}_{r}^{-1 / 2} \mathrm{Nu}=-\left(1+\frac{4}{3} R d\right) \theta^{\prime}(0), \\
& \operatorname{Re}_{r}^{-1 / 2} \mathrm{Sh}=-\phi^{\prime}(0) \quad \text { (active control), }
\end{aligned}
$$

with $\operatorname{Re}_{r}=u_{w} r / \nu_{f}$ representing the Reynolds number at a local point. Conversely, the reduced number of Sherwood for passive control follows the reduced Nusselt number due to the last condition in Eq. (5). We use the term $\mathrm{Nu}_{r}$ for the reduced number of Nusselt and $\mathrm{Sh}_{r}$ for the reduced number of Sherwood.

\section{Solution procedure}

The BVPs of Eqs. (11)-(15) are converted to IVPs by shooting technique. The suitable values for far field boundary condition are guessed, i.e. $\eta \rightarrow \infty$. The first order systems for corresponding Eqs. (11)-(13) are:

$$
\begin{aligned}
& y_{3}^{\prime}=-\left(\frac{n+3}{2}\right) y_{1} y_{3}+n y_{2}^{2}, \\
& y_{5}^{\prime}=\frac{-\left(\frac{n+3}{2}\right) y_{5} y_{1}-N b y_{7} y_{5}-N t y_{5}^{2}}{\frac{1}{\operatorname{Pr}}\left(1+\frac{4}{3} R\right)},
\end{aligned}
$$

$$
y_{7}^{\prime}=-\left(\frac{n+3}{2}\right) \operatorname{Sc} y_{1} y_{7}-\frac{N b}{N t} y_{5}^{\prime}
$$

subject to the conditions at boundaries below:

$$
\begin{aligned}
& y_{1}(0)=0, \quad y_{2}(0)=1, \quad y_{4}(0)=1, \\
& \left\{\begin{array}{l}
y_{6}(0)=1 \\
N b y_{7}(0)+N y_{5}(0)=0 \quad(\text { pastive control } \phi),
\end{array}\right. \\
& y_{2}(\infty) \rightarrow 0, \quad y_{4}(\infty) \rightarrow 0, \quad y_{6}(\infty) \rightarrow 0,
\end{aligned}
$$

where:

$$
\begin{array}{ll}
y_{1}=f, & y_{2}=y_{1}^{\prime}=f^{\prime}, \quad y_{3}=y_{2}^{\prime}=f^{\prime \prime}, \\
y_{4}=\theta, & y_{5}=y_{4}^{\prime}=\theta^{\prime}, \\
y_{6}=\phi, & y_{7}=y_{6}^{\prime}=\phi^{\prime} .
\end{array}
$$

In order to solve Eq. (18), the values for $y_{3}(0)$, i.e. $f^{\prime \prime}(0)$, are not given prior to the computation, but they are required. The calculated value for $f^{\prime \prime}\left(\eta_{\max }\right)=0$ at $\eta_{\max }=12$ (say) is compared with the boundary conditions (21) and (22). In a similar manner, Eqs. (19) and (20) are solved. Next, the initial values of $f^{\prime \prime}(0), \theta^{\prime}(0)$, and $\phi^{\prime}(0)$ are guessed selectively while a MATLAB coding is applied to retrieve the numerical solutions for the model with error tolerance of $10^{-6}$.

\section{Results and discussion}

To get the quantitative results that are stable in terms of consistency and reliability, validation of the present numerical outcomes is performed with available results for various values of $n, N t, \mathrm{Sc}$, and $\mathrm{Pr}$. Based on Table 1, it is found that there is proper agreement between the results of the present paper and the previous paper by Mustafa et al. [23], who employed Keller Box method.

Table 1. Results of comparison between values of $\mathrm{Nu}_{r}$ for contrasting parameters $(\mathrm{Nb}=0.5)$.

\begin{tabular}{cccccc}
\hline \multirow{n}{*}{} & \multirow{N}{*}{$\boldsymbol{N}$} & \multirow{2}{*}{$\mathbf{S c}$} & $\mathbf{P r}$ & \multicolumn{2}{c}{$\mathbf{N u}_{\boldsymbol{r}}$} \\
\cline { 4 - 6 } & & & & $\begin{array}{c}\text { Mustafa } \\
\text { et al. [23] }\end{array}$ & $\begin{array}{c}\text { Present } \\
\text { paper }\end{array}$ \\
\hline 0.5 & 0.5 & 20 & 5 & 1.2170065 & 1.2171088 \\
& 0.7 & & & 0.9815765 & 0.9906997 \\
1.0 & 0.5 & 5 & 5 & 1.6914582 & 1.6915148 \\
& & 10 & & 1.4740787 & 1.4741665 \\
& & 20 & & 1.2861370 & 1.2862569 \\
2.5 & 0.5 & 20 & 0.7 & 0.6619164 & 0.6618473 \\
& & & 5 & 1.4784288 & 1.4786103 \\
& & & 7 & 1.5758736 & 1.5761661 \\
\hline
\end{tabular}


Table 2. Numerical values of $\mathrm{Nu}_{r}$ and $\mathrm{Sh}_{r}$ under nonzero and zero normal fluxes with $\mathrm{Pr}=6, n=1$, and $\mathrm{Sc}=0.5$ $\left(\operatorname{Re}_{r}^{(1 / 2)} C_{f}=-1.17372\right)$.

\begin{tabular}{|c|c|c|c|c|c|c|}
\hline \multirow{2}{*}{$R d$} & \multirow{2}{*}{$N b$} & \multirow{2}{*}{$N t$} & \multicolumn{2}{|c|}{ Nonzero normal flux } & \multicolumn{2}{|c|}{ Zero normal flux } \\
\hline & & & $\mathrm{Nu}_{r}$ & $\mathrm{Sh}_{r}$ & $\mathrm{Nu}_{r}$ & $\mathrm{Sh}_{r}$ \\
\hline 0 & 0.5 & 0.5 & 1.10420 & -0.30454 & 2.40229 & -2.40229 \\
\hline-0.1 & & & 0.96274 & -0.30482 & 2.25404 & -2.60082 \\
\hline-0.2 & & & 0.81176 & -0.29406 & 2.09088 & -2.85120 \\
\hline-0.3 & & & 0.65018 & -0.26338 & 1.90849 & -3.18083 \\
\hline-0.4 & & & 0.47743 & -0.19491 & 1.70003 & -3.64294 \\
\hline \multirow[t]{4}{*}{-0.3} & 0.4 & 0.5 & 0.44509 & 0.14782 & 2.68236 & -5.50949 \\
\hline & 0.5 & & 0.65018 & -0.26338 & 1.90849 & -3.18083 \\
\hline & 0.6 & & 1.32487 & -1.73284 & 1.44365 & -2.00507 \\
\hline & 0.7 & & 4.00767 & -8.32872 & 1.15091 & -1.37013 \\
\hline \multirow[t]{4}{*}{-0.3} & 0.2 & 0.3 & 0.70132 & -0.05149 & 2.60881 & -6.52205 \\
\hline & & 0.4 & 0.47281 & 0.26693 & 2.84395 & -9.47984 \\
\hline & & 0.5 & 0.35698 & 0.38529 & 3.24488 & -13.52032 \\
\hline & & 0.6 & 0.28747 & 0.43992 & 3.56032 & -17.80162 \\
\hline
\end{tabular}

Table 3. Numerical values of $\mathrm{Nu}_{r}$ and $\mathrm{Sh}_{r}$ under nonzero and zero normal fluxes with $\operatorname{Pr}=6, n=1, R d=-0.3$, and $N b=N t=\mathrm{Sc}=0.5$.

\begin{tabular}{cccccc}
\hline \multirow{2}{*}{$\boldsymbol{n}$} & \multicolumn{2}{c}{ Nonzero normal flux } & & \multicolumn{2}{c}{ Zero normal flux } \\
\cline { 2 - 3 } & $\mathbf{N u}_{\boldsymbol{r}}$ & $\mathbf{S h}_{\boldsymbol{r}}$ & & $\mathbf{N u}$ & $\mathbf{S h}_{\boldsymbol{r}}$ \\
\hline 0 & 0.54778 & -0.16121 & & 1.68068 & -2.80113 \\
1 & 0.65018 & -0.26338 & & 1.90850 & -3.18083 \\
2 & 0.73699 & -0.33807 & & 2.11522 & -3.52536 \\
3 & 0.81404 & -0.39926 & & 2.30461 & -3.84101 \\
\hline
\end{tabular}

The calculated values of $\mathrm{Nu}_{r}, \mathrm{Sh}_{r}$, and reduced skin friction coefficients for both nonzero and zero normal fluxes are listed in Tables 2 and 3 with assorted values of $\mathrm{Rd}, \mathrm{Nb}$ and $\mathrm{Nt}$ and with fixed values of $n, \mathrm{Pr}$, and Sc. We can see that heat transfer performance is enhanced under both nonzero and zero normal fluxes as thermal radiation increases. Brownian motion increases the rate of heat transfer under nonzero normal flux, but the effect is reversed under zero normal flux. Oppositely, thermophoresis increases the rate of heat transfer under zero normal flux, but under nonzero normal flux the effect is reversed. On the contrary, both thermal radiation and thermophoresis increase the mass flux under nonzero normal flux, but the effects are opposite under zero normal flux. Contradictorily, the mass flux decreases under nonzero normal flux due to higher Brownian motion, but the effect is opposite under zero normal flux. In addition, rate of heat transfer increases under both nonzero and zero normal fluxes by increasing $n$ values. On the other hand, the mass flux decreases under both nonzero and zero normal fluxes of nanoparticles.

Generally, the $f^{\prime}(\eta)$ profiles in Figures 2-7 are similar for all assorted values of $R, N b, N t, n, \operatorname{Pr}$, and

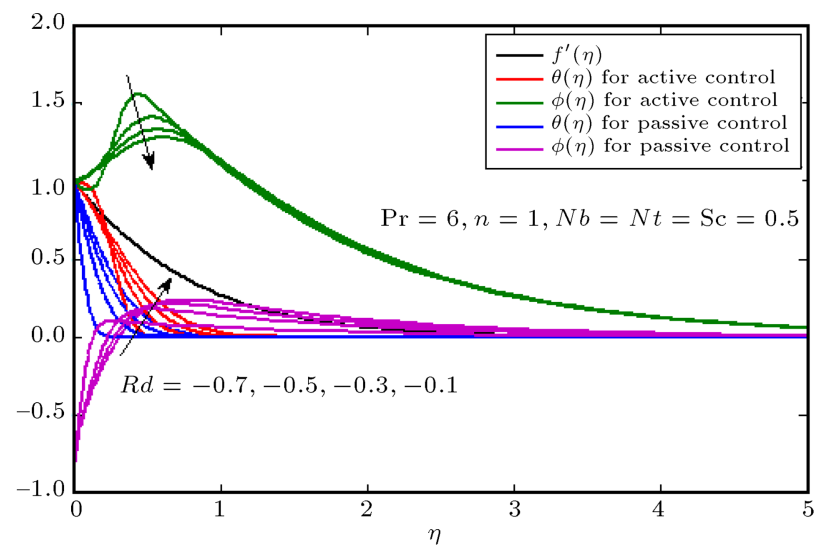

Figure 2. Profiles of $f^{\prime}, \theta$ and $\phi$ of $\eta$ under nonzero and zero normal fluxes for different thermal radiation parameters.

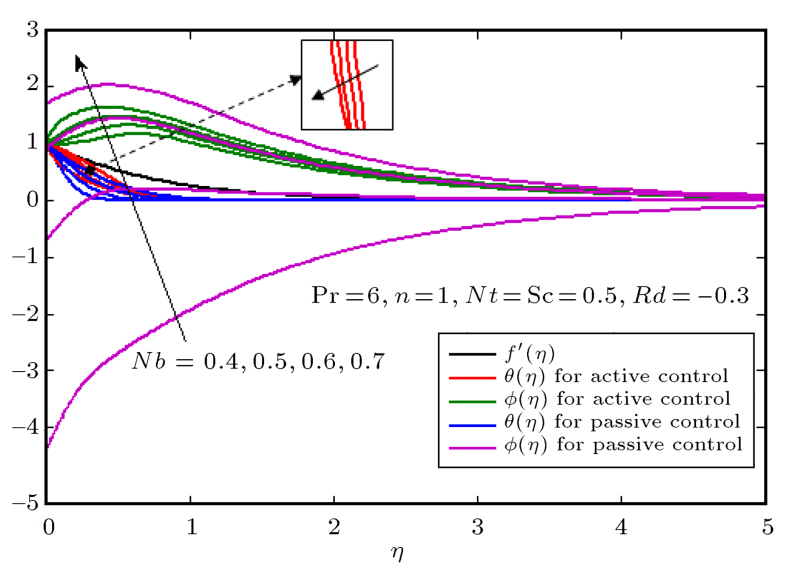

Figure 3. Profiles of $f^{\prime}, \theta$, and $\phi$ of $\eta$ under nonzero and zero normal fluxes for different Brownian motion parameters. 


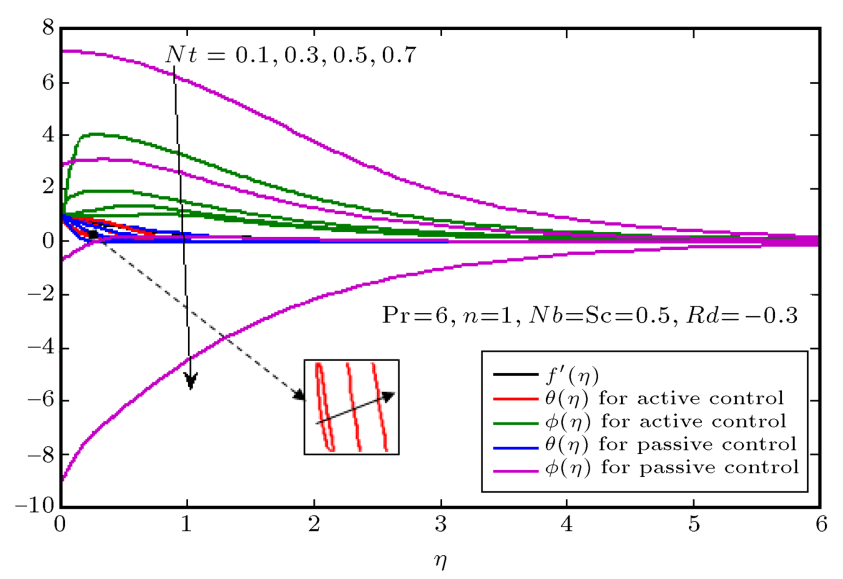

Figure 4. Profiles of $f^{\prime}, \theta$ and $\phi$ of $\eta$ under nonzero and zero normal fluxes for different thermophoresis parameters.

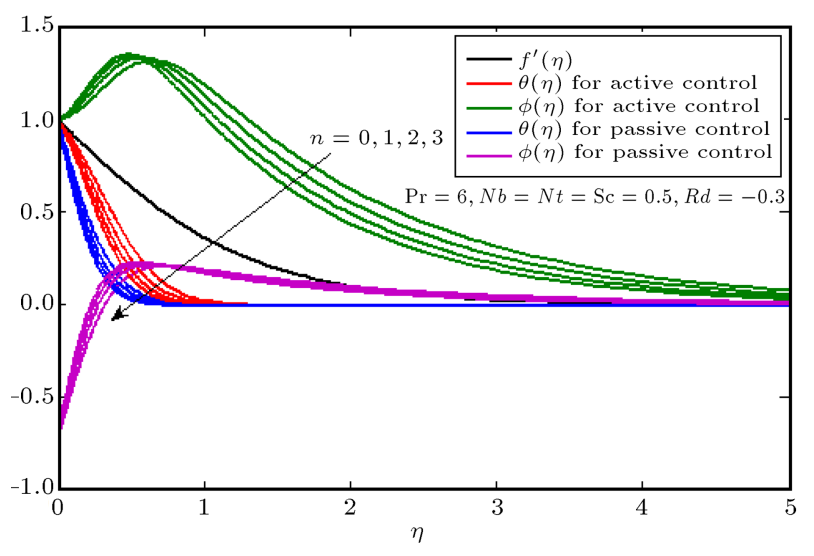

Figure 5. Profiles of $f^{\prime}, \theta$ and $\phi$ of $\eta$ under nonzero and zero normal fluxes for assorted values of index of power law.

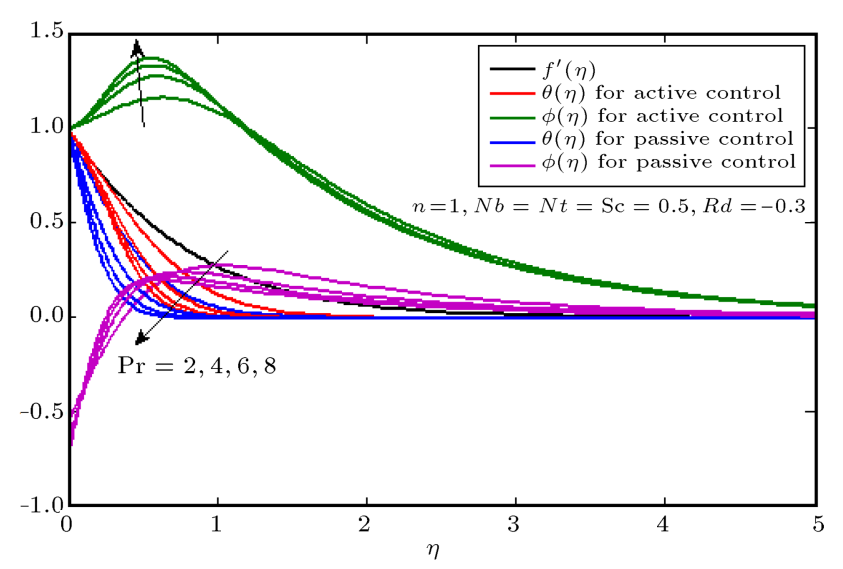

Figure 6. Profiles of $f^{\prime}, \theta$, and $\phi$ of $\eta$ under nonzero and zero normal fluxes for different Prandtl number parameters.

Sc under both nonzero and zero normal fluxes. Clearly, there are changes in $\theta(\eta)$ profile and $\phi(\eta)$ profile for various values of parameters used. A rise in thermal radiation produces an increasing $\phi(\eta)$ profile at first and

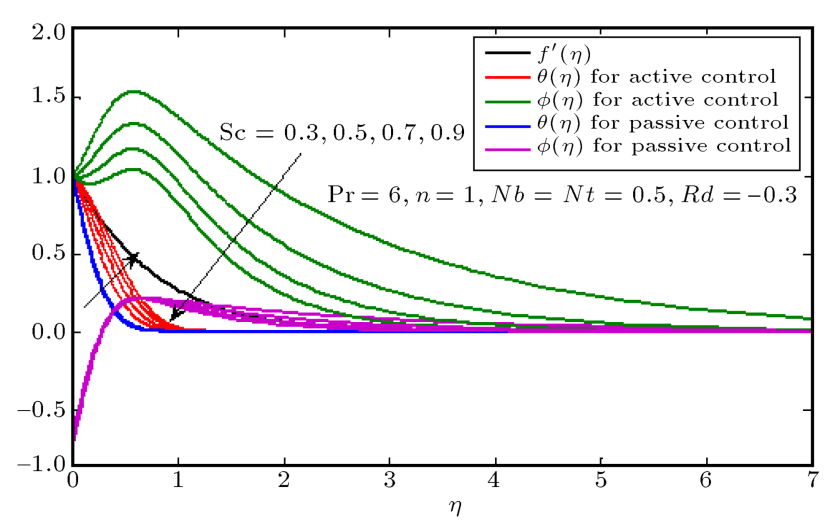

Figure 7. Profiles of $f^{\prime}, \theta$, and $\phi$ of $\eta$ under nonzero and zero normal fluxes for different Schmidt number parameters.

then the profile rapidly decreases for both nonzero and zero normal fluxes. This is because the nanoparticles disperse rapidly by the heat they carry, but as thermal radiation rises, the heat emitted from the nanoparticles also increases and causes the nanoparticles to disperse slowly as time passes. The same goes to the $\theta(\eta)$ profile, where it decreases as the thermal radiation increases by the emitting heat. Brownian motion is a random motion of the nanoparticles when they collide with each other and with the molecules of the fluid. We can see that for nonzero normal flux, the $\theta(\eta)$ profile decreases as the Brownian motion increases and for zero normal flux, the $\theta(\eta)$ profile increases. The $\phi(\eta)$ profile increases as the Brownian motion increases for both nonzero and zero normal fluxes. The $\theta(\eta)$ and $\phi(\eta)$ profiles raise due to collisions between the particles in the nanofluid as a result of enhanced random Brownian motion.

For nonzero normal flux, the $\theta(\eta)$ profile increases as thermophoresis becomes greater, whereas the $\phi(\eta)$ profile decreases. Both $\theta(\eta)$ and $\phi(\eta)$ profiles decrease as the thermophoresis rises for zero normal flux. When the variation of index of power law increases, the $\theta(\eta)$ profile decreases. However, the $\phi(\eta)$ profile increases and then turns into decreasing pattern for both nonzero and zero normal fluxes. As the Prandtl number increases, the $\phi(\eta)$ profile increases and then turns into decreasing pattern for nonzero normal flux. The $\phi(\eta)$ profile decreases, then turns into increasing pattern, and finally returns into decreasing pattern for zero normal flux. The $\theta(\eta)$ profile decreases as Prandtl number increases for both nonzero and zero normal fluxes as growing thermal diffusion rate of the nanofluid causes the temperature to eventually decrease. In both nonzero and zero normal fluxes, as the $\theta(\eta)$ profile increases, Schmidt number also increases. On the other hand, a rise in Schmidt number causes the $\phi(\eta)$ profile to lessen for nonzero normal flux, but for zero normal flux, the $\phi(\eta)$ profile increases at first and then turns into decreasing pattern. 
Some graphical results for $\mathrm{Nu}_{r}$ and $\mathrm{Sh}_{r}$ are included in Figures 8, 9, and 10, respectively. From Figure 8, as the influence of thermic radiation increases, the heat performance under both nonzero and zero normal fluxes enhances, but the effect is reversed for mass flux in both nonzero and zero normal fluxes, in which it decreases. In Figure 9, we can see that the heat

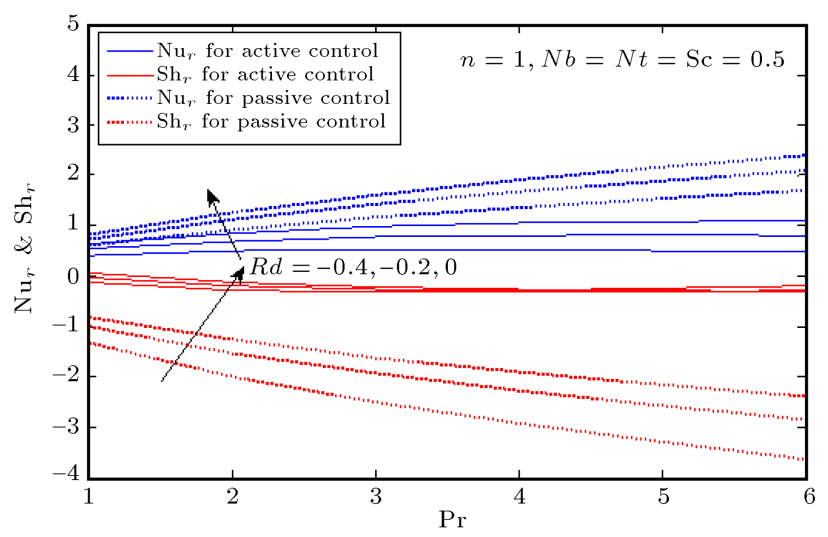

Figure 8. $\mathrm{Nu}_{r}$ and $\mathrm{Sh}_{r}$ under nonzero and zero normal fluxes for different thermal radiation parameters.

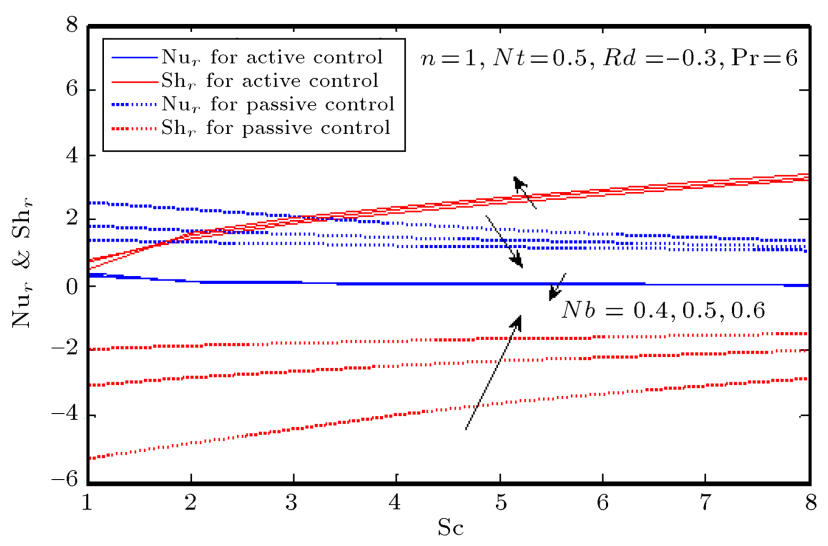

Figure 9. $\mathrm{Nu}_{r}$ and $\mathrm{Sh}_{r}$ under nonzero and zero normal fluxes for different Brownian motion parameters.

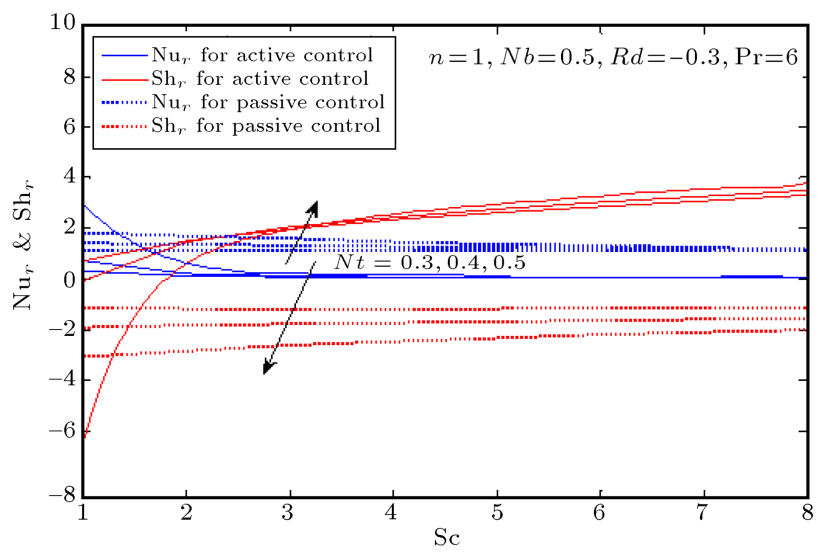

Figure 10. $\mathrm{Nu}_{r}$ and $\mathrm{Sh}_{r}$ under nonzero and zero normal fluxes for different thermophoresis parameters. transfers under both nonzero and zero normal fluxes decrease as Brownian motion increases. Oppositely, the mass fluxes under both nonzero and zero normal fluxes increase as Brownian motion increases. Figure 10 tells us that the mass transfer under nonzero normal flux decreases as thermophoresis increases, but the effect is opposite under zero normal flux. The mass flux under nonzero normal flux is enhanced as thermophoresis is enhanced, but under zero normal flux the effect acts oppositely.

\section{Conclusion}

An axisymmetric boundary layer flow saturated with homogenous nanoparticles under influence of both zero and nonzero normal fluxes is firstly considered in this present work. The governed equations consist of continuity, conservative momentum, energy, and volumetric fraction of nanoparticles under regular assumptions of boundary layer. The energy equation is extended to include thermal radiation effect on the heat transfer of the boundary layer flow over a radially stretching surface. Then, the governed equations are reformed into a set of non-linear ODEs by reduction in similarity equations. The numerical findings are retrieved via a shooting approach by using MATLAB. Several $f^{\prime}(\eta)$, $\theta(\eta)$, and $\phi(\eta)$ profiles under nonzero and zero normal fluxes are presented graphically. The consequent effects on the reduced coefficients of skin friction, $\mathrm{Nu}_{r}$, and $\mathrm{Sh}_{r}$ are also studied. From the results, we can conclude that thermal radiation, Brownian random motion, and thermophoretic diffusion have significant influences on the temperature rundown and the nanoparticles volumetric fraction of the flow concentration. The following conclusions are highlighted:

- Thermal radiation improves heat transfer performance of the axisymmetric flow for both cases of nonzero and zero normal fluxes of nanoparticles;

- With greater Prandtl number, the heat transfer rate of zero normal flux also increases;

- Thermophoresis slightly improves the flow heat transfer performance for the case of nanoparticles with zero normal flux.

\section{Acknowledgement}

The contributing authors thank the MOHE, Malaysia, for the research grant FGRS/1/2015/SG04/ UM/02/1(Project No. FP016-2015A).

\section{References}

1. Choi, S.U.S. and Eastman, J.A. "Enhancing thermal conductivity of fluids with nanoparticles", ASME International Mechanical Engineering Congress \& Exposition, 231, pp. 99-105 (1995). 
2. Gorla, R.S.R. and Kumari, M. "Mixed convection in an axisymmetric stagnation flow of a non-Newtonian nanofluid on a vertical cylinder", Journal of Nanoengineering and Nanosystems, 227(3), pp. 150-160 (2012).

3. Noghrehabadi, A., Pourrajab, R. and Ghalambaz, M. "Effect of partial slip boundary condition on the flow and heat transfer of nanofluid past stretching sheet prescribed constant wall temperature", International Journal of Thermal Sciences, 54, pp. 253-261 (2012).

4. Tamim, H., Dinarvand, S., Hosseini, R., Khalili, S. and Khalili, A. "Mixed convection boundary-layer flow of a nanofluid near stagnation-point on a vertical plate with effect of buoyancy assisting and opposing flows", Research Journal of Applied Sciences, Engineering and Technology, 6(10), pp. 1785-1793 (2013).

5. Buongiorno, J. "Convective transport in nanofluid", ASME, 128, pp. 240-250 (2006).

6. Ariel, P.D. "Axisymmetric flow due to a stretching sheet with partial slip", International Journal of Computers and Mathematics with Applications, 54, pp. 1169-1183 (2007).

7. Sajid, M., Ahmad, I., Hayat, T. and Ayub, M. "Series solution for unsteady axisymmetric flow and heat transfer over a radially stretching sheet", Communication in Nonlinear Science and Numerical Simulation, 13, pp. 2193-2202 (2008).

8. Grosan, T. and Pop, I. "Axisymmetric mixed convection boundary layer flow past a vertical cylinder in a nanofluid", International Journal of Heat and Mass Transfer, 54, pp. 3139-3145 (2011).

9. Lok, Y.Y., Merkin, J.H. and Pop, I. "Mixed convection flow near the axisymmetric stagnation point on a stretching or shrinking cylinder", International Journal of Thermal Sciences, 59, pp. 186-194 (2012).

10. Vajravelu, K., Prasad, K.V. and Santhi, S.R. "Axisymmetric magneto-hydrodynamic (MHD) flow and heat transfer at a non-isothermal stretching cylinder", Applied Mathematics and Computation, 219, pp. 39934005 (2012).

11. Das, K., Duari, P.R. and Kundu, P.K. "Nanofluid flow over an unsteady stretching surface in presence of thermal radiation", Alexandria Engineering Journal, 53, pp. 737-745 (2014).

12. Sajid, M. and Hayat, T. "Influence of thermal radiation on the boundary layer flow due to an exponentially stretching sheet", International Communications in Heat and Mass Transfer, 35, pp. 347-356 (2008).

13. Siddiqa, S., Hossain, M.A. and Saha, S.C. "The effect of thermal radiation on the natural convection boundary layer flow over a wavy horizontal surface", International Journal of Thermal Science, 84, pp. 143150 (2014).

14. Ul Haq, R., Nadeem, S., Khan, Z.H. and Akbar, N.S. "Thermal radiation and slip effects on MHD stagnation point flow of nanofluid over a stretching sheet", Physica E, 65, pp. 17-23 (2015).
15. Abdul Hakeem, Kalaivanan, R., Ganesh, N.V. and Ganga, B. "Effect of partial slip on hydromagnetic flow over a porous stretching sheet with non-uniform heat source/sink, thermal radiation and wall mass transfer", Ain Shams Engineering Journal, 5, pp. 913-922 (2014).

16. Abdul Hakeem, Ganesh, N.V. and Ganga, B. "Magnetic fields effect on second order slip flow of nanofluid over a stretching/shrinking sheet with thermal radiation effect", Journal of Magnetism and Magnetic Materials, 381, pp. 243-257 (2015).

17. Hassani, M., Mohammad Tabar, M., Nemati, H., Domairry, G. and Noori, F. "An analytical solution for boundary layer flow of a nanofluid past a stretching sheet", International Journal of Thermal Sciences, 50, pp. 2256-2263 (2011).

18. Makinde, O.D. and Aziz, A. "Boundary layer flow of a nanofluid past a stretching sheet with a convective boundary condition", International Journal of Thermal Sciences, 50, pp. 1326-1332 (2011).

19. Mustafa, M., Hayat, T., Pop, I., Asghar, S. and Obaidat, S. "Stagnation-point flow of a nanofluid towards a stretching sheet", International Journal of Heat and Mass Transfer, 54, pp. 5588-5594 (2011).

20. Nadeem, S., Ul Haq, R. and Khan, Z.H. "Heat transfer analysis of water-based nanofluid over an exponentially stretching sheet", Alexandria Engineering Journal, 53, pp. 219-224 (2014).

21. Akbar, N.S., Khan, Z.H. and Nadeem, S. "The combined effect of slip and convective boundary condition on stagnation-point flow of CNT suspended nanofluid over a stretching sheet", Journal of Molecular Liquids, 196, pp. 21-25 (2014).

22. Ganesh, N.V., Abdul Hakeem, and Ganga, B. "A comparative theoretical study on $\mathrm{Al}_{2} \mathrm{O}_{3}$ and $\gamma-\mathrm{Al}_{2} \mathrm{O}_{3}$ nanoparticles with different base fluids over a stretching sheet", Advanced Powder Technology, 27(2), pp. 436-441 (2014).

23. Mustafa, M., Khan, J.A., Hayat, T. and Alsaedi, A. "Analytical and numerical solution foe axisymmetric flow of nanofluid due to non-linearly stretching sheet", International Journal of Non-Linear Mechanics, 71, pp. 22-29 (2015).

24. Kostoglou, M. "The equimaterial approach for the numerical solution of the one dimensional transient diffusion equation with zero flux boundary condition", Applied Mathematics and Computation, 218, pp. 13531359 (2011).

25. Burger, R., Frid, H. and Karlsen, K.H. "On the wellposedness of entropy solution to conversation laws with zero-flux boundary condition", Journal of Mathematical Analysis and Applications, 326, pp. 108-120 (2007).

\section{Biographies}

Najwa Ahda Ramly received her first pre-university certificate at College Mara Malacca (KMM) in 2010. Then, she continued her studies at the Universiti 
Malaysia Terengganu (UMT) and received the BSc in Computational Mathematics in 2013 with her finalyear project on "System Dynamics Model Approach on Obesity in Malaysia". Currently, she is working for MSc in Mathematics at University of Malaya (UM) with her thesis entitled "Active and Passive Controls of Convective Flow and Heat Transfer of Nanofluid Over A Surface". She is expecting graduation in 2017.

Sivasankaran Sivanandam received his MSc, MPhil, and $\mathrm{PhD}$ degrees from Bharathiar University, India. After that, he received Post-Doctoral Fellowship from National Cheng Kung University, Taiwan, and National Taiwan University, Taiwan. He was a research professor at Yonsei University, South Korea. Also, he was an Assistant Professor at Sungkyunkwan University, South Korea. Presently, he is a senior lecturer in the Institute of Mathematical Sciences, University of Malaya, Malaysia. He is a member of editorial board in several international journals and reviewer of more than 30 international journals. He is an Associate Editor in the Journal of Applied Fluid Mechanics \& Frontiers in Mechanical Engineering. His areas of interest are convective heat and mass transfer, CFD, nanofluids, micro-channel heat sinks, and porous media.

Noor Fadiya Mohd Noor joined Institute of Mathematical Sciences, University of Malaya (UM), as a senior lecturer in 2012. Prior to that, she was a senior lecturer in the Department of Mechatronics, Faculty of Engineering, Universiti Selangor (UNISEL), Malaysia. In 2010, she obtained her PhD in Numerical Methods from Universiti Kebangsaan Malaysia (UKM) while working full time in UNISEL since 2005. Known as an active reviewer in more than 40 ISI-indexed journals, yearly, she is interested in multidisciplinary research areas, which include numerical methods in fluid mechanics, nanofluid boundary layer flows, $\mathrm{C}++$ programming, and profit equalization reserve in Islamic banking. 